\title{
In Situ Observations of Solar Wind Stream Interface Evolution
}

\author{
K.D.C. Simunac • L.M. Kistler • A.B. Galvin • M.A. Lee • M.A. Popecki • C. Farrugia • \\ E. Moebius · L.M. Blush • P. Bochsler • P. Wurz • B. Klecker • \\ R.F. Wimmer-Schweingruber • B. Thompson • J.G. Luhmann • C.T. Russell • \\ R.A. Howard
}

Received: 8 December 2008 / Accepted: 3 June 2009 / Published online: 30 June 2009

(C) The Author(s) 2009. This article is published with open access at Springerlink.com

\begin{abstract}
The heliocentric orbits of the two STEREO satellites are similar in radius and ecliptic latitude, with separation in longitude increasing by about $45^{\circ}$ per year. This arrangement provides a unique opportunity to study the evolution of stream interfaces near $1 \mathrm{AU}$ over time scales of hours to a few days, much less than the period of a Carrington rotation. Assuming nonevolving solar wind sources that corotate with the Sun, we calculated the expected time and longitude of arrival of stream interfaces at the Ahead observatory based
\end{abstract}

L.M. Blush now in Prilly, Switzerland.

STEREO Science Results at Solar Minimum

Guest Editors: Eric R. Christian, Michael L. Kaiser, Therese A. Kucera, O.C. St Cyr.

K.D.C. Simunac $(\varangle) \cdot$ L.M. Kistler · A.B. Galvin · M.A. Lee · M.A. Popecki · C. Farrugia ·

E. Moebius · P. Bochsler

University of New Hampshire Space Science Center, Durham, NH 03824, USA

e-mail: ksimunac@ cisunix.unh.edu

L.M. Blush · P. Bochsler · P. Wurz

Physikalisches Institut, University of Bern, Bern, Switzerland

B. Klecker

Max-Planck Institut für Extraterrestrische Physik, Garching, Germany

R.F. Wimmer-Schweingruber

Institute for Experimental and Applied Physics, University of Kiel, Kiel, Germany

B. Thompson

NASA Goddard Space Flight Center, Greenbelt, MD 20771, USA

J.G. Luhmann

Space Sciences Laboratory, University of California, Berkeley, CA 94720, USA

C.T. Russell

Institute of Geophysics and Planetary Physics, University of California, Los Angeles, CA 90095, USA

R.A. Howard

E.O. Hulburt Center for Space Research, Naval Research Laboratory, Washington, DC 20375, USA 
on the in situ solar wind speeds measured at the Behind observatory. We find agreement to within $5^{\circ}$ between the expected and actual arrival longitude until the spacecraft are separated by more than $20^{\circ}$ in heliocentric inertial longitude. This corresponds to about one day between the measurement times. Much larger deviations, up to $25^{\circ}$ in longitude, are observed after $20^{\circ}$ separation. Some of the deviations can be explained by a latitude difference between the spacecraft, but other deviations most likely result from evolution of the source region. Both remote and in situ measurements show that changes at the source boundary can occur on a time scale much shorter than one solar rotation. In 32 of 41 cases, the interface was observed earlier than expected at STEREO/Ahead.

\section{Solar Wind Stream Interfaces}

From the earliest in situ observations the solar wind was considered to have a bimodal structure; that is, it was divided into two basic populations: slow and fast. Neugebauer and Snyder (1966) first described the average properties of the solar wind based on in situ data from Mariner 2, launched in 1962. Fast-moving, hot streams (up to $830 \mathrm{~km} \mathrm{~s}^{-1}$ and $9 \times 10^{5} \mathrm{~K}$ ) alternate with slower streams having lower temperatures (down to about $300 \mathrm{~km} \mathrm{~s}^{-1}$ and $3 \times 10^{4} \mathrm{~K}$ ). The fast streams are seen to recur with the Sun's equatorial rotation period of about 27 days. At the leading edge of the fast streams are density enhancements, but outside of these times the density and velocity are inversely related.

It is generally agreed that fast solar wind originates from coronal holes (e.g., Krieger, Timothy, and Roelof, 1973), whereas the slow solar wind comes from the vicinity of the streamer belt. Under the assumption of a dipolar magnetic field aligned with the Sun's rotation axis, a stationary observer in the ecliptic plane will see an alternating pattern of slow and fast wind because the Sun's axis of rotation is tilted approximately $7^{\circ}$ with respect to the ecliptic plane.

The transition from slow to fast solar wind is called a "stream interface" (SI), a term coined by Burlaga in 1974. Burlaga (1974) defined a SI as follows: "At 1 AU there is a distinct boundary ... characterized by an abrupt (approximately a factor of 2 change in $<10^{6} \mathrm{~km}$ ) drop in density, a similar increase in temperature, and a small increase in speed." A superposed epoch analysis by Gosling et al. (1978) provided further characterization of SIs. They observed that SIs follow 2 to 36 hours after a magnetic field polarity reversal. The SI separates dense, slow-moving plasma from fast, less dense plasma. In the corotating reference frame, there is a velocity shear at the SI, which decreases with radial distance from the Sun as momentum is transferred across the SI. The electron temperature rises by about $40 \%$, and the alpha particle abundance usually increases. Gosling et al. (1978) suggested that the fast and slow solar wind streams have different origins. This was supported by later studies such as that of Wimmer-Schweingruber, von Steiger, and Paerli (1997), which showed that solar wind composition changed with the passage of a SI. In addition, Gosling et al. (1978) also reported that the plasma in the vicinity of a SI undergoes an east - west deflection in the ecliptic plane. A similar deflection in the out-of-ecliptic (north-south) plane confirmed that SIs are really three-dimensional structures (Gosling et al., 1993).

Although there have been many observations of SIs, multipoint observations are sparse except in the vicinity of L1. Nolte and Roelof (1973a, 1973b) used observations from Pioneer 8, Pioneer 9, and Vela to test the mapping of interplanetary structures back to the Sun, including a case with a high-speed SI. Neugebauer et al. (1998) combined measurements from the Ulysses and Wind spacecraft to determine the latitudinal dependencies of structures in the solar wind and their mapping to the corona using different source surface 
models for solar activity minimum time periods. Neugebauer et al. (2002) extended this analysis to active times, using data from the Ulysses and ACE spacecraft.

During the minimum and rising phases of solar cycle 21, the Helios 1 and 2 spacecraft provided measurements from 0.3 to 1 AU. These data were combined with observations made near 1 AU. Schwenn (1990) reports that SIs have Parker-spiral-like geometry. Schwenn fit a single Archimedean (Parker) spiral through two observation points and used this to calculate an effective radial SI propagation speed as

$$
V_{\text {eff }}=\Omega_{\text {Sun }}\left(R_{2}-R_{1}\right) /\left(\varphi_{2}-\varphi_{1}\right),
$$

where $\Omega_{\text {Sun }}$ is the Sun's angular speed, $R_{1}$ and $R_{2}$ are the orbital radii of the two observation points, and $\varphi_{1}$ and $\varphi_{2}$ are the longitudes of the observers in the corotating Carrington frame. If the two observation points are not separated in either radius or longitude, then the effective speed is undefined. The separation in Carrington longitude between the STEREO observatories increases with time for the period studied here, but the radial separation waxes and wanes between 0.03 and $0.13 \mathrm{AU}$.

With several dozen interaction regions observed by both STEREO spacecraft at solar minimum, this study will test the scale under which basic assumptions of Parker-spiral geometry are valid near $1 \mathrm{AU}$. The two primary assumptions are ideal corotation of the SI with the Sun and negligible source evolution. If these assumptions hold it should be possible to predict the arrival time and location of a SI at one observatory after it has been observed at the other by using the mathematical definition of an Archimedean spiral. In addition, the effective propagation speed as calculated by Schwenn (1990) is expected to fall within the range of observed solar wind speeds at $1 \mathrm{AU}$.

\section{The STEREO/PLASTIC Proton Data Set}

The Solar Terrestrial Relations Observatory (STEREO) was successfully launched in October 2006. STEREO is composed of two nearly identical spacecraft in heliocentric orbit. STEREO/Ahead (STEREO/A) has an orbit just inside 1 AU, and STEREO/Behind (STEREO/B) is just outside $1 \mathrm{AU}$. The different radii result in orbital periods shorter than one year for STEREO/A and longer than one year for STEREO/B. The heliographic longitude separation between the two observatories increases by about $45^{\circ}$ per year. For a more detailed description of the STEREO mission see Kaiser et al. (2008). STEREO was launched near the minimum of the solar activity cycle, so the in situ observations of the solar wind have been dominated by recurring patterns of slow and fast solar wind with very few transients. This has been a nearly ideal circumstance to study the transitions between slow and fast solar wind streams. The data used in this study are primarily proton data from the Plasma and Suprathermal Ion Composition (PLASTIC) investigation, described in detail by Galvin et al. (2008).

The proton parameters are calculated by using a one-dimensional velocity distribution function created from a single coincidence rate on a minute-by-minute basis. A Maxwellian distribution function is fit to the measured distribution function to obtain density, bulk speed, and 1-D temperature for the solar wind protons. The data are then averaged over 10-minute intervals. These bulk proton parameters are used to identify SIs, as described in the next section. 
Figure 1 Proton bulk

parameters from PLASTIC/A.

Top panel is bulk proton speed, middle panel is proton density, and bottom panel is proton thermal speed. Data have been 10 minute averaged. The SI is indicated with shading.

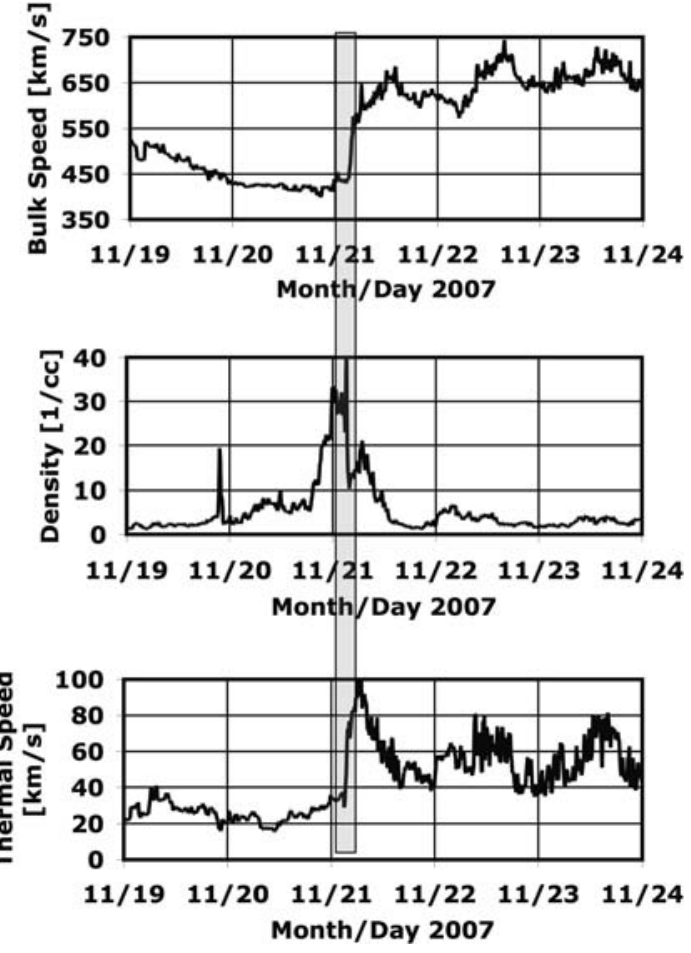

\section{Observations}

Figure 1 shows a sample SI observed by PLASTIC/A. The SI was identified following the criteria set by Burlaga (1974): an increase in speed accompanied by a drop in density and an increase in temperature. Using these sharp parameter variations we are able to time the SI crossing to within one hour. We have identified in this manner 41 SIs that were observed by both spacecraft between March 2007 and February 2008 (Carrington Rotations 2054 through 2066). Table 1 lists the SI arrival times at STEREO/B, the solar wind speed measured at that time by PLASTIC/B, the time difference between arrivals at the two observatories (with time differences less than zero indicating that STEREO/A was the first observer), the latitude and longitude separations of the observatories in Heliocentric Inertial (HCI) coordinates, the difference in orbital radii in AU, the difference between the expected and actual longitude of arrival at STEREO/A, and the difference between the expected and actual arrival time at STEREO/A. In the HCI coordinate system $z$ is the solar rotation axis, and $x$ is the Sun's ascending node in the ecliptic plane. In other words, the HCI coordinate system does not corotate with the Sun. Latitude differences less than zero indicate that STEREO/A is south of STEREO/B.

The expected longitude of SI arrival at STEREO/A is calculated by assuming the SI is an ideal Archimedean spiral that is propagating at the solar wind speed measured by PLASTIC/B. Any effects from the difference in latitude between the observatories are neglected. The calculation is made in the Carrington coordinate system, using the photospheric equatorial angular speed $\Omega_{\text {Sun }}=14.38^{\circ}$ s per day (Newton and Nunn, 1951):

$$
\varphi_{\mathrm{A}_{\text {expected }}}=\varphi_{\mathrm{B}}-\Omega_{\mathrm{Sun}}\left(R_{\mathrm{B}}-R_{\mathrm{A}}\right) / V_{\mathrm{BSW}} .
$$


Table 1 Summary list of stream interfaces.

\begin{tabular}{|c|c|c|c|c|c|c|c|c|}
\hline No. & $\begin{array}{l}\text { Interface arrival } \\
\text { time at B (UT) }\end{array}$ & $\begin{array}{l}V_{\mathrm{sw}} \text { at } \mathrm{B} \\
\left(\mathrm{km} \mathrm{s}^{-1}\right)\end{array}$ & $\begin{array}{l}\text { Time } \\
\text { from B } \\
\text { to A } \\
\text { (hours) }\end{array}$ & $\begin{array}{l}\Delta \mathrm{HCI} \\
\text { latitude } \\
\text { (degrees) }\end{array}$ & $\begin{array}{l}\Delta \mathrm{HCI} \\
\text { longitude } \\
\text { (degrees) }\end{array}$ & $\begin{array}{l}\Delta R \\
(\mathrm{AU})\end{array}$ & $\begin{array}{l}\text { Expected- } \\
\text { observed } \\
\text { Carrington } \\
\text { longitude at } \\
\text { A (degrees) }\end{array}$ & $\begin{array}{l}\text { Expected- } \\
\text { observed } \\
\text { arrival } \\
\text { time at A } \\
\text { (hours) }\end{array}$ \\
\hline 1 & $\begin{array}{l}5 \text { March } 2007 \\
02: 05\end{array}$ & 370 & -2.58 & -0.07 & 1.4 & 0.039 & -0.2 & -0.4 \\
\hline 2 & $\begin{array}{l}6 \text { March } 2007 \\
\text { 11:00 }\end{array}$ & 445 & -3.25 & -0.06 & 1.5 & 0.040 & -1.0 & -1.9 \\
\hline 3 & $\begin{array}{l}\text { 11 March } 2007 \\
\text { 23:45 }\end{array}$ & 385 & -3.92 & 0.00 & 1.7 & 0.043 & -1.1 & -1.9 \\
\hline 4 & $\begin{array}{l}\text { 12 March } 2007 \\
14: 40\end{array}$ & 500 & -1.00 & 0.01 & 1.8 & 0.044 & -0.1 & -0.3 \\
\hline 5 & $\begin{array}{l}\text { 25 March } 2007 \\
08: 15\end{array}$ & 360 & -8.08 & 0.18 & 2.5 & 0.053 & -3.3 & -6.0 \\
\hline 6 & $\begin{array}{l}\text { 1 April } 2007 \\
04: 00\end{array}$ & 400 & -1.42 & 0.29 & 3.1 & 0.058 & -0.2 & -0.3 \\
\hline 7 & $\begin{array}{l}9 \text { April } 2007 \\
\text { 10:15 }\end{array}$ & 310 & -2.58 & 0.43 & 3.8 & 0.065 & 0.1 & 0.1 \\
\hline 8 & $\begin{array}{l}22 \text { April } 2007 \\
\text { 18:40 }\end{array}$ & 365 & -2.75 & 0.72 & 5.1 & 0.076 & -1.4 & -2.6 \\
\hline 9 & $\begin{array}{l}23 \text { April } 2007 \\
05: 15\end{array}$ & 380 & -3.00 & 0.73 & 5.1 & 0.076 & -1.2 & -3.2 \\
\hline 10 & $\begin{array}{l}27 \text { April } 2007 \\
\text { 16:00 }\end{array}$ & 500 & 0.83 & 0.83 & 5.6 & 0.080 & -1.2 & -2.2 \\
\hline 11 & $\begin{array}{l}7 \text { May } 2007 \\
15: 15\end{array}$ & 425 & -0.50 & 1.08 & 6.9 & 0.088 & -2.0 & -3.7 \\
\hline 12 & $\begin{array}{l}\text { 18 May } 2007 \\
07: 10\end{array}$ & 380 & 7.50 & 1.36 & 8.3 & 0.096 & 2.1 & 4.0 \\
\hline 13 & $\begin{array}{l}2 \text { June } 2007 \\
17: 30\end{array}$ & 405 & 1.33 & 1.75 & 10.7 & 0.107 & -3.4 & -6.3 \\
\hline 14 & $\begin{array}{l}\text { 22 June } 2007 \\
\text { 02:00 }\end{array}$ & 445 & 3.00 & 2.12 & 14.2 & 0.118 & -5.9 & -10.8 \\
\hline 15 & $\begin{array}{l}29 \text { June } 2007 \\
14: 30\end{array}$ & 420 & 16.75 & 2.20 & 15.6 & 0.122 & 0.8 & 1.5 \\
\hline 16 & $\begin{array}{l}3 \text { July } 2007 \\
18: 25\end{array}$ & 530 & 18.33 & 2.23 & 16.4 & 0.123 & -0.6 & -1.1 \\
\hline 17 & $\begin{array}{l}\text { 10 July } 2007 \\
20: 45\end{array}$ & 390 & 14.25 & 2.23 & 17.8 & 0.126 & -2.0 & -3.7 \\
\hline 18 & $\begin{array}{l}\text { 14 July } 2007 \\
11: 10\end{array}$ & 460 & 18.33 & 2.22 & 18.6 & 0.127 & -1.7 & -3.1 \\
\hline 19 & $\begin{array}{l}\text { 20 July } 2007 \\
04: 45\end{array}$ & 375 & 21.42 & 2.16 & 19.8 & 0.128 & 0.4 & 0.8 \\
\hline 20 & $\begin{array}{l}\text { 26 July } 2007 \\
13: 25\end{array}$ & 385 & 15.67 & 2.05 & 21.1 & 0.129 & -4.2 & -7.7 \\
\hline
\end{tabular}


Table 1 (Continued)

\begin{tabular}{|c|c|c|c|c|c|c|c|c|}
\hline No. & $\begin{array}{l}\text { Interface arrival } \\
\text { time at B (UT) }\end{array}$ & $\begin{array}{l}V_{\mathrm{sw}} \text { at } \mathrm{B} \\
\left(\mathrm{km} \mathrm{s}^{-1}\right)\end{array}$ & $\begin{array}{l}\text { Time } \\
\text { from B } \\
\text { to A } \\
\text { (hours) }\end{array}$ & $\begin{array}{l}\Delta \mathrm{HCI} \\
\text { latitude } \\
\text { (degrees) }\end{array}$ & $\begin{array}{l}\Delta \mathrm{HCI} \\
\text { longitude } \\
\text { (degrees) }\end{array}$ & $\begin{array}{l}\Delta R \\
(\mathrm{AU})\end{array}$ & $\begin{array}{l}\text { Expected- } \\
\text { observed } \\
\text { Carrington } \\
\text { longitude at } \\
\text { A (degrees) }\end{array}$ & $\begin{array}{l}\text { Expected- } \\
\text { observed } \\
\text { arrival } \\
\text { time at A } \\
\text { (hours) }\end{array}$ \\
\hline 21 & $\begin{array}{l}29 \text { July } 2007 \\
07: 15\end{array}$ & 450 & 4.58 & 1.99 & 21.7 & 0.129 & -12.0 & -22.0 \\
\hline 22 & $\begin{array}{l}6 \text { August } 2007 \\
04: 40\end{array}$ & 330 & 26.83 & 1.77 & 23.3 & 0.129 & 1.1 & 2.0 \\
\hline 23 & $\begin{array}{l}\text { 15 August } 2007 \\
14: 10\end{array}$ & 470 & 18.83 & 1.40 & 25.3 & 0.128 & -8.2 & 14.9 \\
\hline 24 & $\begin{array}{l}24 \text { August } 2007 \\
\text { 23:00 }\end{array}$ & 410 & 44.17 & 0.93 & 27.2 & 0.126 & 4.0 & 8.4 \\
\hline 25 & $\begin{array}{l}31 \text { August } 2007 \\
18: 10\end{array}$ & 350 & 26.83 & 0.52 & 28.6 & 0.124 & -5.1 & -9.2 \\
\hline 26 & $\begin{array}{l}\text { 14 September } 2007 \\
\text { 10:50 }\end{array}$ & 370 & 19.50 & -0.41 & 31.2 & 0.119 & -12.6 & -23.3 \\
\hline 27 & $\begin{array}{l}20 \text { September } 2007 \\
00: 25\end{array}$ & 435 & 45.92 & -0.83 & 32.2 & 0.116 & -0.4 & -0.8 \\
\hline 28 & $\begin{array}{l}26 \text { September } 2007 \\
19: 30\end{array}$ & 430 & 48.92 & -1.36 & 33.4 & 0.112 & -0.1 & -0.2 \\
\hline 29 & $\begin{array}{l}3 \text { October } 2007 \\
03: 30\end{array}$ & 540 & 29.17 & -1.87 & 34.4 & 0.108 & -13.5 & -24.6 \\
\hline 30 & $\begin{array}{l}17 \text { October } 2007 \\
08: 30\end{array}$ & 440 & 47.67 & -3.00 & 36.6 & 0.099 & -4.9 & -8.9 \\
\hline 31 & $\begin{array}{l}24 \text { October } 2007 \\
21: 00\end{array}$ & 515 & 34.50 & -3.57 & 37.6 & 0.093 & -14.2 & -25.9 \\
\hline 32 & $\begin{array}{l}13 \text { November } 2007 \\
10: 40\end{array}$ & 480 & 23.00 & -4.81 & 39.9 & 0.078 & -23.2 & -42.4 \\
\hline 33 & $\begin{array}{l}20 \text { November } 2007 \\
06: 50\end{array}$ & 410 & 20.92 & -5.14 & 40.6 & 0.073 & -24.7 & -45.1 \\
\hline 34 & $\begin{array}{l}24 \text { November } 2007 \\
06: 50\end{array}$ & 510 & 41.67 & -5.30 & 40.9 & 0.070 & -14.7 & -26.7 \\
\hline 35 & $\begin{array}{l}9 \text { December } 2007 \\
20: 00\end{array}$ & 530 & 68.33 & -5.66 & 42.3 & 0.058 & -2.1 & -3.8 \\
\hline 36 & $\begin{array}{l}\text { 16 December } 2007 \\
\text { 11:00 }\end{array}$ & 490 & 60.50 & -5.68 & 42.7 & 0.054 & -6.8 & -12.4 \\
\hline 37 & $\begin{array}{l}\text { 18 December } 2007 \\
\text { 11:40 }\end{array}$ & 520 & 59.00 & -5.67 & 42.9 & 0.052 & -8.0 & -14.7 \\
\hline 38 & $\begin{array}{l}3 \text { January } 2008 \\
18: 40\end{array}$ & 450 & 78.50 & -5.30 & 43.9 & 0.043 & 1.5 & 2.7 \\
\hline
\end{tabular}


Table 1 (Continued)

\begin{tabular}{|c|c|c|c|c|c|c|c|c|}
\hline No. & $\begin{array}{l}\text { Interface arrival } \\
\text { time at B (UT) }\end{array}$ & $\begin{array}{l}V_{\mathrm{sw}} \text { at } \mathrm{B} \\
\left(\mathrm{km} \mathrm{s}^{-1}\right)\end{array}$ & $\begin{array}{l}\text { Time } \\
\text { from B } \\
\text { to A } \\
\text { (hours) }\end{array}$ & $\begin{array}{l}\Delta \mathrm{HCI} \\
\text { latitude } \\
\text { (degrees) }\end{array}$ & $\begin{array}{l}\Delta \mathrm{HCI} \\
\text { longitude } \\
\text { (degrees) }\end{array}$ & $\begin{array}{l}\Delta R \\
(\mathrm{AU})\end{array}$ & $\begin{array}{l}\text { Expected- } \\
\text { observed } \\
\text { Carrington } \\
\text { longitude at } \\
\text { A (degrees) }\end{array}$ & $\begin{array}{l}\text { Expected- } \\
\text { observed } \\
\text { arrival } \\
\text { time at A } \\
\text { (hours) }\end{array}$ \\
\hline 39 & $\begin{array}{l}\text { 10 January } 2008 \\
16: 10\end{array}$ & 435 & 85.33 & -5.00 & 44.3 & 0.040 & 4.8 & 8.7 \\
\hline 40 & $\begin{array}{l}30 \text { January } 2008 \\
02: 20\end{array}$ & 360 & 80.83 & -3.72 & 45.2 & 0.035 & 1.4 & 2.6 \\
\hline 41 & $\begin{array}{l}9 \text { February } 2008 \\
10: 00\end{array}$ & 465 & 57.50 & -2.82 & 45.7 & 0.034 & -12.4 & -22.6 \\
\hline
\end{tabular}

The time corresponding to the expected arrival longitude is then obtained by using the STEREO orbit tool (available at http://stereo-ssc.nascom.nasa.gov/where/).

From March 2007 through February 2008 the HCI longitude separation between the STEREO observatories grew from less than $2^{\circ}$ to just over $48^{\circ}$. The separation in HCI latitude varied but never exceeded $6^{\circ}$. The difference in orbital radii varied from 0.03 to 0.13 AU. The temporal separation between SI observations ranged from 10 minutes to 3.5 days, allowing us to study the evolution of SIs over periods much shorter than a complete solar rotation.

To illustrate the differences in the in situ data between the two spacecraft, Figure 2(a) shows the proton bulk speed from STEREO/A and STEREO/B for Carrington Rotations 2061 through 2064, covering approximately mid-September through December 2007. The identified SIs for the two spacecraft are marked with vertical lines (red for STEREO/A and blue for STEREO/B) and labeled with the numbers corresponding to their listings in Table 1. The longitudinal separation between STEREO/A and STEREO/B during this time increases from $33^{\circ}$ to $45^{\circ}$. Assuming a rigid corotation rate of $14.38^{\circ}$ per day, and a typical garden hose angle of $45^{\circ}$ at $1 \mathrm{AU}$, we would expect the time difference between observations is to increase from about 45 to about 73 hours. (These values are less than the longitude difference divided by the Sun's angular speed because of the small difference in orbital radii between the observatories.) The actual time differences ranged from 21 hours (SI no. 33) to 68 hours (SI no. 35).

To compare the data between observatories in more detail, we have plotted proton bulk speed versus back-mapped Carrington longitude in Figure 2(b). In the "ballistic" backmapping technique it is assumed the solar wind has traveled with constant speed radially outward from the solar source surface, as described by Nolte and Roelof (1973a). The orbital radius of the spacecraft is divided by the measured solar wind speed to obtain a travel time. The Sun's equatorial angular speed is divided by the travel time to obtain the difference in longitude between the source and the observatory. This method of estimating the source longitude has been used in previous studies including those by Nolte and Roelof (1973a, 1973b) and Neugebauer et al. $(1998,2002)$. The error in the source longitude calculated using this technique is estimated to be $\leq 10^{\circ}$ (Nolte and Roelof, 1973a). Note that in Figure 2(b) the back-mapped slow-to-fast solar wind transitions are stretched across a wide range of longitudes compared to the real-time in situ measurements in Figure 2(a). This is not unexpected; material from a range of longitudes at the source is compressed as the fast solar wind tries to overtake the slow wind in corotating interaction regions (CIRs). 

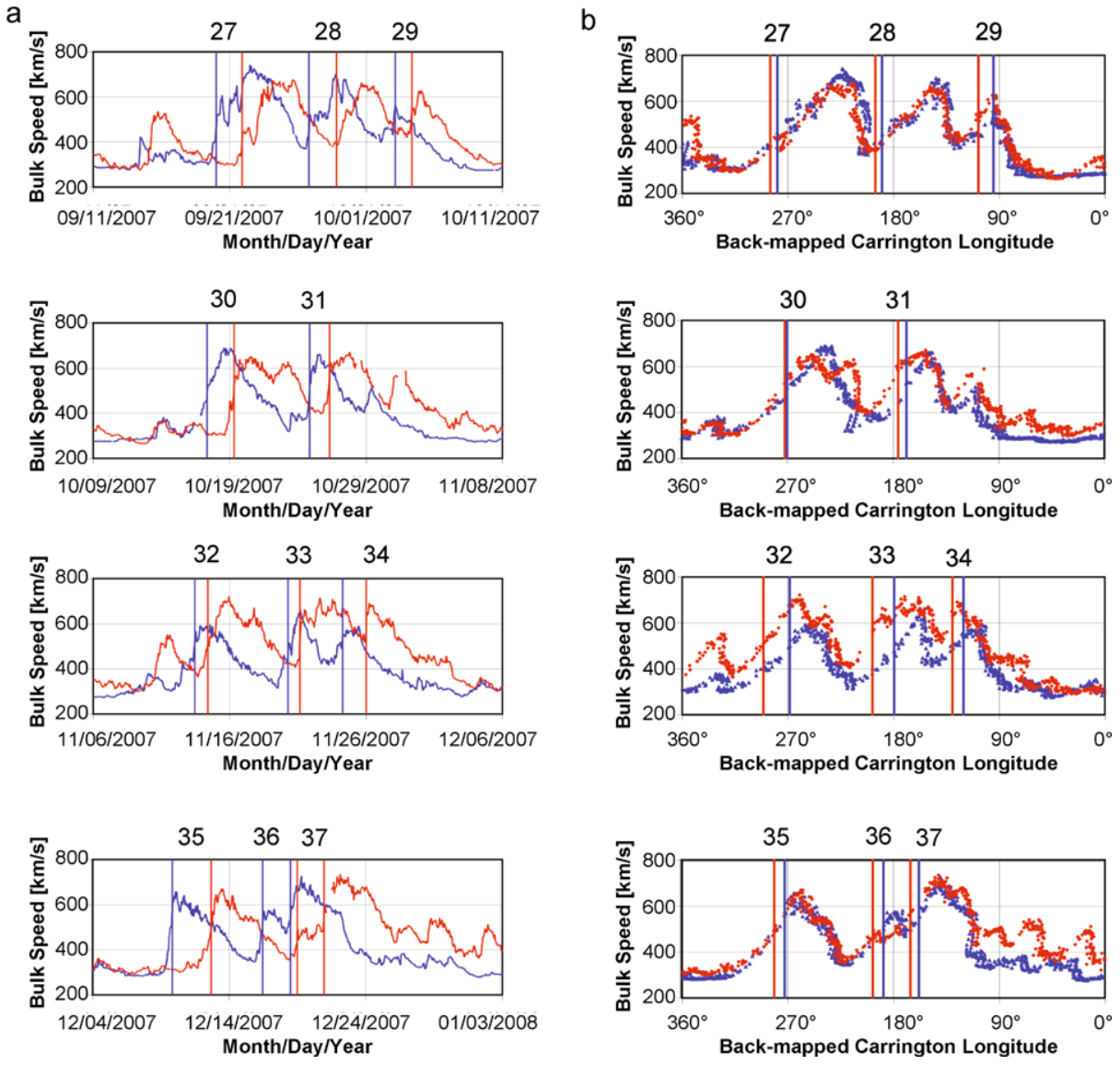

Figure 2 Proton bulk speed versus time (a) and back-mapped Carrington longitude (b) for Carrington Rotations 2061 through 2064. The red trace is STEREO/A, and the blue trace is STEREO/B. Vertical bars indicate the positions of SIs. Note that the back-mapped Carrington longitudes are reversed from typical synoptic maps.

Under the assumptions of perfect corotation and negligible source evolution, the backmapped speed profiles in Figure 2(b) are expected to match between the two observatories. Recurring streams (and their associated CIRs) should be observed at nearly the same Carrington longitudes for successive solar rotations under the same assumptions. Vertical lines in Figure 2(b) again indicate the SIs identified for this study. Note that although only the solar wind speed is shown, there are additional density and temperature criteria for SI identification. As a result, even when speeds are the same for both spacecraft, the back-mapped longitude of the SIs may not agree, such as for SI no. 35.

In this back-mapped representation, the large- and small-scale features of each Carrington rotation can be compared. In Figure 2 each Carrington rotation begins and ends with slow solar wind. The middle of each rotation is dominated by fast solar wind with three high-speed peaks and short periods of slower wind between them. The high-speed peaks move slightly from right to left with each Carrington rotation, indicating they recur sooner than expected (again under the assumption that they corotate with the Sun's equatorial an- 
gular speed of $14.38^{\circ}$ per day). This early recurrence could be explained by growth of the source and/or by movement of the source with respect to the photosphere.

In the top panel of Figure 2(b), Carrington Rotation (CR) 2061, the first two highspeed streams (corresponding to SI nos. 27 and 28) show very similar speed profiles for STEREO/A and STEREO/B. The back-mapped longitudinal extent is the same for both observatories. The maximum observed solar wind speed is about $60 \mathrm{~km} \mathrm{~s}^{-1}$ greater at STEREO/B than at STEREO/A. The back-mapped longitudes of the SIs agree within $6^{\circ}$ between the two observatories. The third SI, no. 29, shows more differences between the two observatories. SI 29 back-maps to $108^{\circ}$ Carrington longitude from STEREO/A and to $95^{\circ}$ Carrington longitude from STEREO/B. The high-speed stream following SI 29 backmaps to a region covering $10^{\circ}$ more longitude from PLASTIC/A than from PLASTIC/B. The maximum observed solar wind speed is about $630 \mathrm{~km} \mathrm{~s}^{-1}$ at $\mathrm{A}$ and $560 \mathrm{~km} \mathrm{~s}^{-1}$ at B. The observatories were separated by about $33^{\circ}$ in HCI longitude and by about $2^{\circ}$ in $\mathrm{HCI}$ latitude.

In CR 2062, the first SI (no. 30) maps to nearly identical longitudes $\left(271^{\circ}\right.$ and $\left.272^{\circ}\right)$ from the two spacecraft, but the speed profiles following the SI are obviously different. PLASTIC/A records two high-speed peaks, but PLASTIC/B saw just one. The second transition (SI no. 31) maps back to about $176^{\circ}$ from $\mathrm{A}$ and to $169^{\circ}$ from $\mathrm{B}$. The high-speed stream is about $10^{\circ}$ wider in back-mapped longitude from STEREO/A than from STEREO/B. The third transition from slow to fast solar wind is not marked with a SI, nor is it given a number in Table 1 because the SI criteria of a simultaneous drop in density and increase in temperature accompanying an increase in speed were not met.

The Carrington rotation in Figure 2 with the greatest apparent discrepancies in speed profiles and SI longitudes between Ahead and Behind is CR 2063 (November 2007), when HCI longitudinal separation was about $40^{\circ}$ and HCI latitude separation was about $5^{\circ}$. By CR 2064 (bottom panel of Figure 2) the speed profiles are showing better agreement again. The longitudinal separation between the observatories for CR 2064 increased to about $43^{\circ}$, and the latitudinal separation was about $5^{\circ}$. Possible explanations for the different speed profiles in CR 2063 include the following: movement of the source (i.e., nonideal corotation), evolution of the source (such as shrinking or growing surface area), which can result in a change in the radial speed at which material leaves the source (cf. Nolte et al., 1976; Wang and Sheeley, 1990), and back-mapping to different source latitudes at the Sun. According to the STEREO/IMPACT level 3 event list (available at http://www-ssc.igpp.ucla.edu/forms/stereo/stereo_level_3.html), no interplanetary coronal mass ejections were observed during CR 2063, so the possibility of transient events will not be explored.

We begin by examining the sources of the fast streams: the coronal holes. Some coronal holes rotate rigidly, independent of the photosphere (see, for example, Timothy, Krieger, and Vaiana, 1975). Others have latitude-dependent differential rotation similar to the photosphere (see, for example, Wang and Sheeley, 1993). Nolte et al. (1976) showed that the speed of the solar wind emanating from a coronal hole is proportional to the area of the hole within $10^{\circ}$ of the ecliptic plane. Similarly, Wang and Sheeley (1990) showed that the total coronal hole area is correlated with observed solar wind speeds at 1 AU. Figure 3 shows synoptic plots of modeled coronal holes obtained from the Global Oscillation Network Group (GONG) for the same Carrington rotations as Figure 2. To create these plots, a potential-field source surface model with the source surface fixed at 2.5 solar radii $\left(R_{\text {Sun }}\right)$ is determined from hourly synoptic solar magnetograms. Purple and orange shading represent regions of open fields (i.e., coronal holes) with opposite magnetic polarity. The solid black curve is the modeled location of the heliospheric current sheet (HCS). Green lines indicate 
fields that are closed out to just less than $2.5 R_{\text {Sun }}$. In terms of Carrington longitude, observation time goes from right to left. In other words, a feature observed at CR 2063.25 would be found at $270^{\circ}$ Carrington longitude on the synoptic map for CR 2063. In each synoptic map there is an equatorial coronal hole extending from the northern pole in the vicinity of longitudes $225^{\circ}$ to $270^{\circ}$. There is a broader coronal hole extending from the southern pole between about $80^{\circ}$ and $180^{\circ}$ longitude. Both regions are shaded in Figure 3. Note that although the large-scale features are the same, the boundaries of the holes do change from one solar rotation to the next. The equatorward extent of the modeled southern coronal hole moves to the right (west), crossing the edge of the shaded region in CR 2064. Similarly, the equatorward extent of the modeled northern hole also moves to the right between CR 2061 and 2062.

Figures 4, 5, 6 and 7 show the same GONG modeled coronal hole maps, combined with plots of back-mapped solar wind speed measured by PLASTIC. The in situ data are plotted in time from right to left for easier comparison with the synoptic maps. The middle two panels of Figures 4 through 7 show the back-mapped radial magnetic field polarity measured by STEREO/IMPACT (see Luhmann et al., 2008). The bottom two panels are synoptic maps created from $195 \AA$ wavelength extreme ultraviolet (EUV) observations from STEREO/SECCHI (see Howard et al., 2008), with Ahead images above and Behind images below. Regions of open magnetic field in the EUV images appear darker than closed fields, indicating the locations of coronal holes. The images from both STEREO/A and STEREO/B can be compared with the modeled coronal holes on the GONG synoptic maps. The synoptic EUV images can also be compared with each other to look for changes that have taken place during the interval between observations at the two observatories. Comparing images between the two observatories should be done with caution; the apparent size of the disk and the viewing angles are different between the two observatories. Also note that the time at which the image at a particular longitude is taken does not always correspond to the back-mapped time the material measured in situ left the source region. The nearly horizontal tracks across the GONG maps in Figures 4 through 7 are projections of the spacecraft trajectories. Vertical lines again indicate the back-mapped longitudes of the SIs, and the numbers correspond to Table 1.

In Figures 4 through 7 the fast solar wind comes from the longitudes corresponding to the coronal holes, as expected. The right-most velocity peak corresponds to the northern coronal hole. The middle peak corresponds to the first encounter with the southern coronal hole. There is a velocity minimum, followed by the left-most peak corresponding to a second encounter with the southern coronal hole. We will concentrate our analysis on the middle peaks (SI nos. 28, 31, 33, and 36), as they show clear SIs between slow and fast solar wind. We will first examine whether latitude dependence can explain the observed changes. We will then look in more detail at source motion and evolution.

If the SI at the Sun is not vertical (i.e., oriented north-south), then the slope of the SI combined with the latitudinal separation between the observatories can result in an apparent longitudinal offset in the observations. Leske et al. (2008) and Mason et al. (2009) note the importance of latitude separation when comparing CIR energetic particle data between the STEREO observatories. This idea is illustrated in Figure 8 and was described before by Lee (2000). Because the SI slope is not remotely observed, a potential proxy is the slope of the HCS at the longitude where it was crossed. (The GONG consortium models the neutral line at the source surface.) As Gosling et al. (1978) noted, SIs are generally observed several hours after a current sheet crossing. We tried this technique to correct for latitude separation with mixed results.

Both STEREO observatories crossed the current sheet just before encountering SI no. 28 in the middle of CR 2061. The slope of the line tangent to the modeled current sheet is about 
Figure 3 GONG synoptic coronal hole maps for Carrington Rotations 2061 through 2064. The orange and purple shading denote opposite polarities (orange for positive and purple for negative). The green lines are magnetic field lines that are closed at the model's source surface. White areas represent field lines that close below the source surface radius of $2.5 R_{\text {Sun }}$. The solid black curve is the heliospheric current sheet.
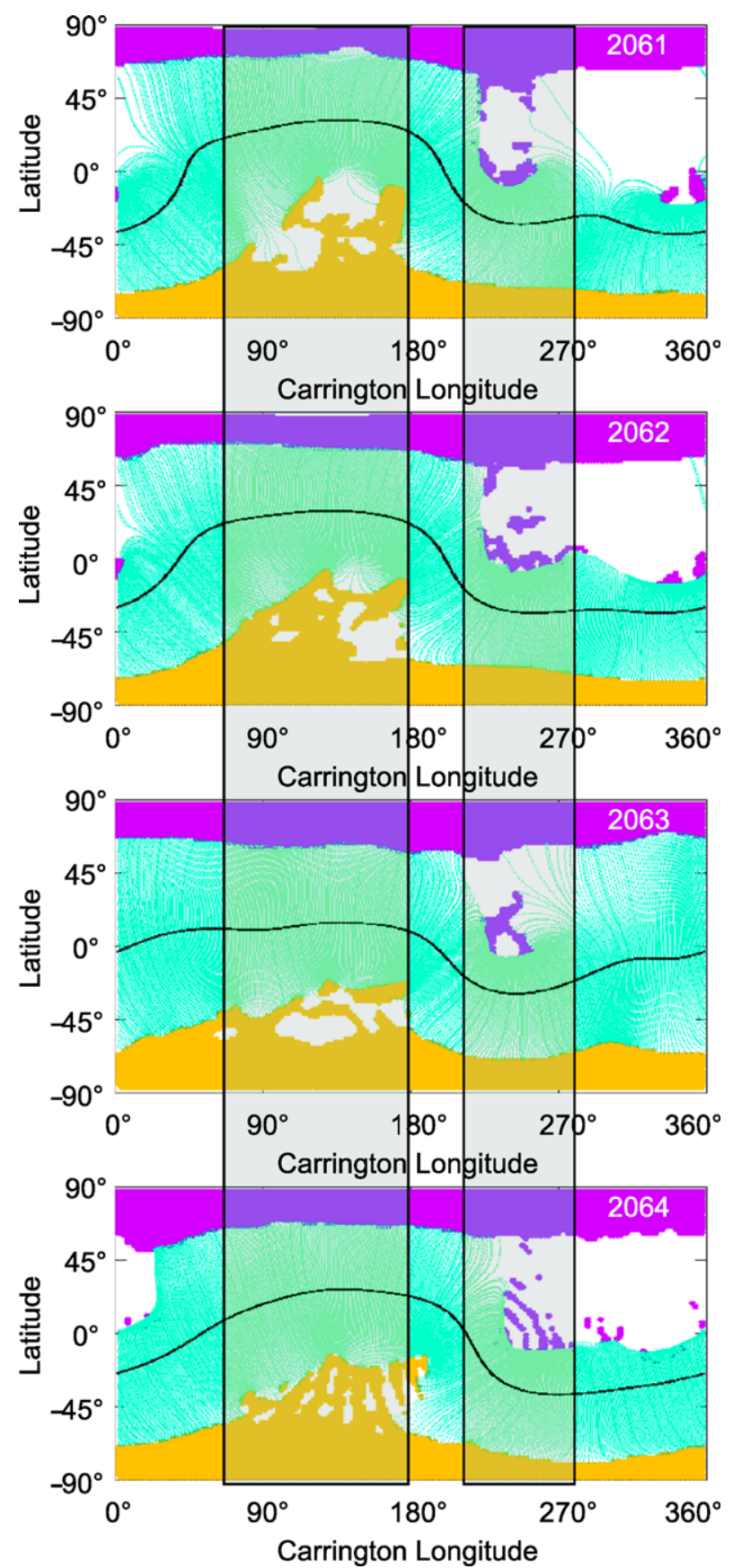

-1.80 . The observatories were separated by $1.5^{\circ} \mathrm{HCI}$ latitude at this time. The extra backmapped longitude $(\Delta \varphi)$ that would have to be covered by spacecraft B before encountering 
Figure 4 The top panel is the observed solar wind speed back-mapped to its source longitude. The second panel is the GONG synoptic plot of modeled coronal holes and the modeled neutral line for Carrington Rotation 2061. The horizontal red and blue traces are the projections of the STEREO spacecraft trajectories. The middle panels are the sign of the radial component of the magnetic field observed by

STEREO/IMPACT and mapped back to the source longitude in the same manner as the proton bulk speed. The bottom two panels are $195 \AA$ SECCHI observations, with SECCHI-A above and SECCHI-B below. Vertical lines indicate the back-mapped longitudes of the SIs, with numbers corresponding to Table 1 . Note that time goes from right to left in all of the plots.
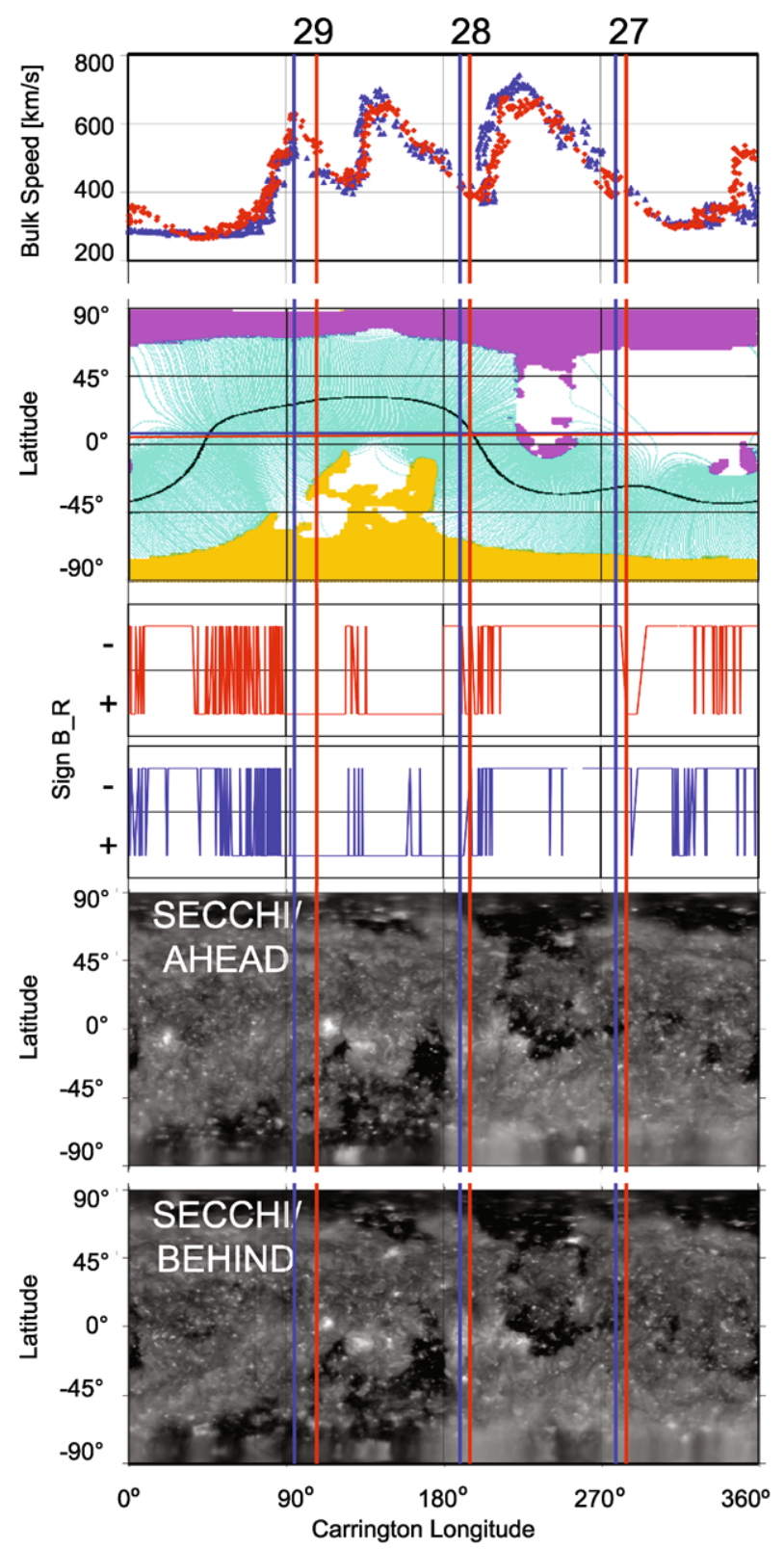

the SI is $7.3^{\circ}$ is calculated following Lee (2000) as

$$
\Delta \varphi=\frac{\Omega_{\mathrm{Sun}}\left(R_{\mathrm{B}}-R_{\mathrm{A}}\right)}{V_{\mathrm{sw}}}+\frac{\theta_{\mathrm{A}}-\theta_{\mathrm{B}}}{\text { slope }} .
$$

The actual offset in back-mapped Carrington longitude is $5.6^{\circ}$.

During CR 2062 the observatories crossed the current sheet at about $200^{\circ}$ back-mapped Carrington longitude, just before encountering SI no. 31. The slope of the modeled neutral 
Figure 5 Same as Figure 4, but for Carrington Rotation 2062.

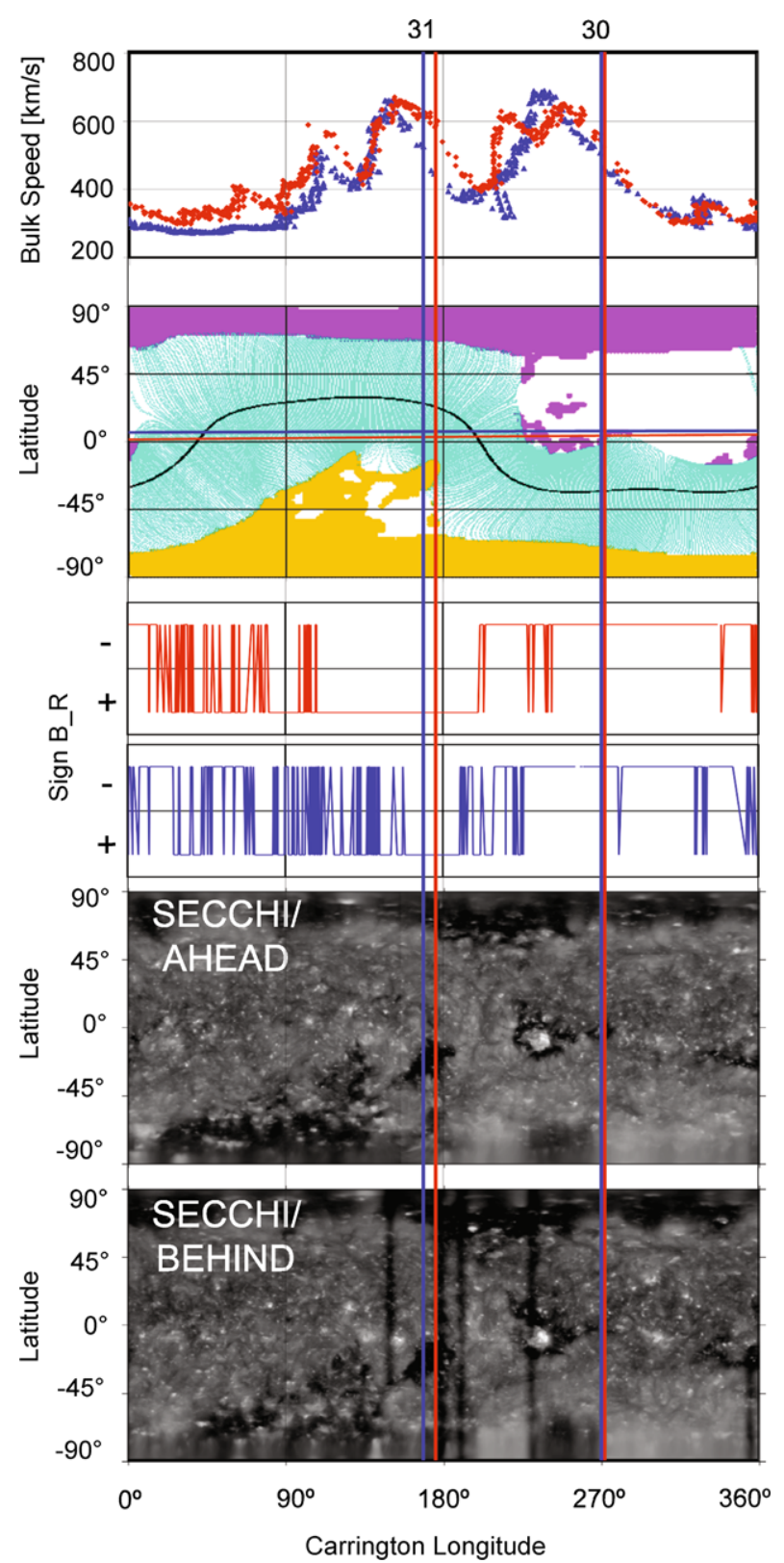

line is approximately -1.68 . The expected offset in longitude is $6.8^{\circ}$. The actual offset in back-mapped Carrington longitude is $7.1^{\circ}$, which agrees well with the calculated value. In this case using the slope of the modeled neutral line (in place of a real SI slope) seems to work.

One Carrington rotation later (2063) the slope of the modeled current sheet has changed such that it is less steep, about -1.00 . The HCI latitude separation between the two spacecraft has increased to about $5.25^{\circ}$. The same calculation as before yields an expected longi- 
Figure 6 Same as Figure 4, but for Carrington Rotation 2063.

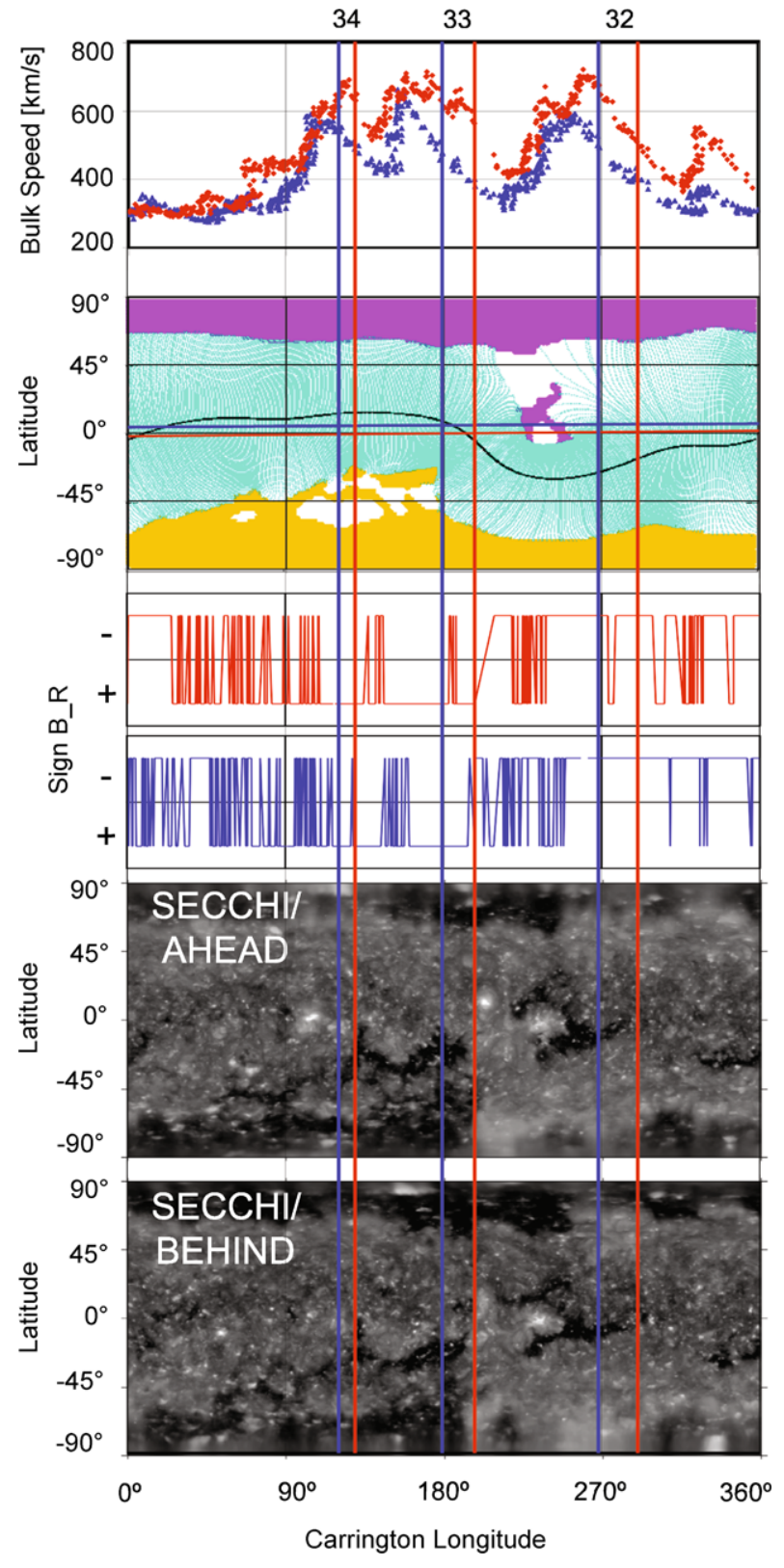

tude offset of $9.6^{\circ}$. The actual offset in back-mapped Carrington longitude for SI no. 33 is about $18.4^{\circ}$, almost twice the calculated value. The SI slope would need to be 0.37 to obtain the larger offset, which is not an unreasonable value. However, from CR 2061 through 2064 the slope of the modeled neutral line only varies from -1.0 to -1.82 . Either latitude separation is not the only cause for the offset in back-mapped longitude or the neutral line slope is not a good substitute for the SI slope in this case. 
Figure 7 Same as Figure 4, but for Carrington Rotation 2064.

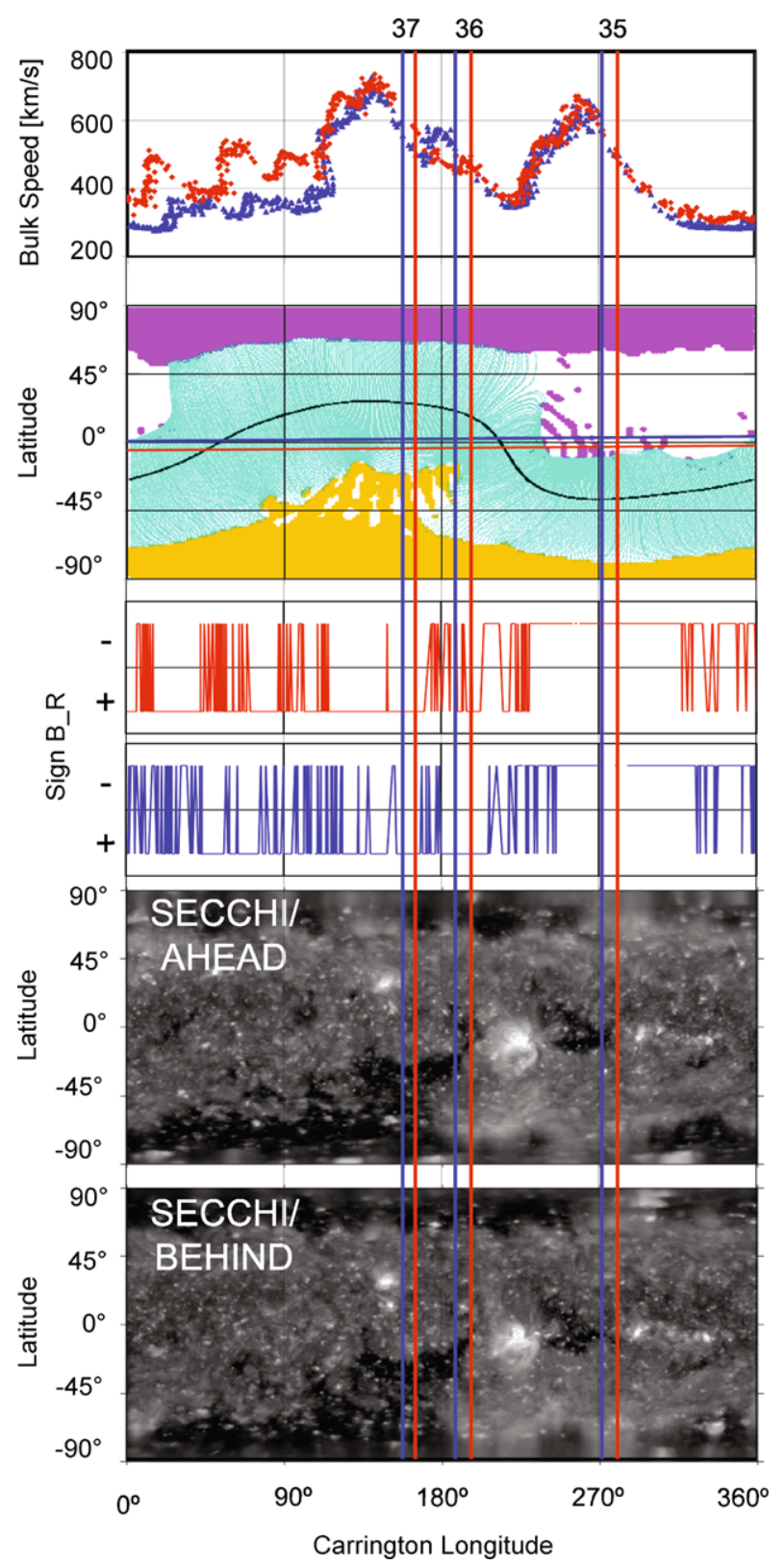

Finally, the modeled current sheet prior to SI no. 36 in the middle of CR 2064 has a slope of about -1.82 , similar to the slope during CR 2062, but steeper than in CR 2063. The expected offset in back-mapped Carrington longitude is $6.0^{\circ}$. The actual offset is $9.0^{\circ}$.

Another possible reason for the timing difference between the spacecraft (also related to latitude separation) is the latitude of the current sheet. A spacecraft on the positive-polarity side of the current sheet would not see the high-speed flow from a coronal hole with negative polarity. In the case of SI no. 33, the current sheet is so near to the ecliptic plane that 

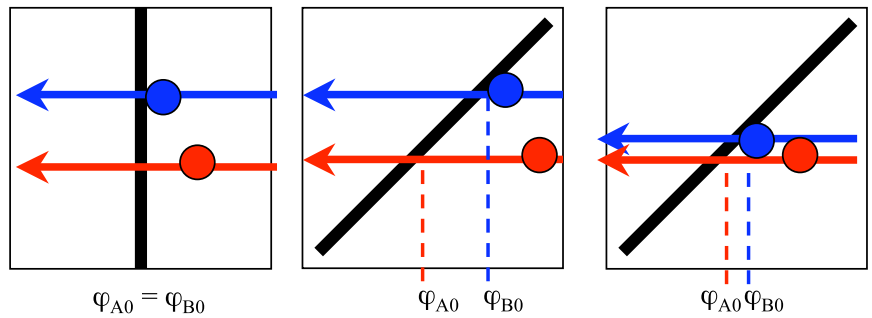

Figure 8 Simple illustration to demonstrate the importance of the slope of the SI when the two observatories are separated in latitude. Each panel shows the out-of-ecliptic plane orthogonal to the Sun - observer line. The SI is the solid black line. The projected spacecraft trajectories are the red and blue arrows.

the GONG model shows it may fall between the spacecraft from Carrington longitudes of $180^{\circ}$ to $190^{\circ}$ (see the second panel of Figure 6). However, plotting the radial magnetic field polarity recorded by IMPACT (the middle two panels of Figure 6), we see that the observed radial magnetic field polarity at both spacecraft reverses at about $200^{\circ}$. So the solar wind observed by spacecraft B maps to the correct side of the current sheet to observe the coronal hole material, thus excluding current sheet location as a possible explanation for the disparity in back-mapped SI longitude. Note that the right-hand (western) boundary of the southern coronal hole in the SECCHI maps extends to larger Carrington longitudes than the modeled coronal hole in the GONG map, suggesting the model does not agree well with observations. This may explain why the correction for latitude separation did not work in the second example here.

We find that latitudinal differences may explain some of the differences in the in situ data between the two spacecraft. However, in some cases, particularly in the case of SI no. 33, the time differences are larger than expected. We now look at how the source is changing during these time periods to determine whether that also plays a role.

In CR 2061 (Figure 4), the velocity profile for the middle peak, corresponding to the southern coronal hole and SI no. 28, is very similar between spacecraft A and spacecraft B. SI no. 28 was back-mapped to $195^{\circ}$ Carrington longitude from STEREO/A and to $190^{\circ}$ from STEREO/B. The SI was observed shortly after a region of mixed polarity, indicating current sheet material. There is reasonable agreement between the back-mapped current sheet crossing longitude of about $200^{\circ}$ Carrington longitude and the predicted longitude from the GONG modeled current sheet. The SECCHI synoptic images show coronal holes with the same locations and boundaries as the GONG model indicates. There are no obvious differences between the SECCHI/A and SECCHI/B images. The HCI latitudinal separation of the two spacecraft in this case was $1.5^{\circ}$.

In CR 2062 (Figure 5), the difference between the STEREO/A and STEREO/B backmapped longitudes of SI no. 31 (the recurrence of SI no. 28) has increased to about $8^{\circ}$. As mentioned earlier, the high speed observed by STEREO/A back-maps to a larger range of Carrington longitudes than from STEREO/B. The latitude separation between the spacecraft is $3.7^{\circ}$. The top two panels in Figure 9 shows the $195 \AA$ observations from SECCHI at the times corresponding to the SI departing the source surface (based on ballistic backmapping) during CR 2062. The images have been scaled so that the apparent disk size is the same for both observatories. A qualitative comparison of the images from STEREO/B and STEREO/A show the boundaries of the coronal hole do not appear to change substantially in the two days between SECCHI/B and SECCHI/A observations.

The top panel in Figure 6 shows that the peak speed observed by PLASTIC/A is larger than the peak speed observed by PLASTIC/B by $50 \mathrm{~km} \mathrm{~s}^{-1}$ following SI no. 33. The Nolte 
Figure 9 SECCHI $195 \AA$ images corresponding to the times when the SIs 31,33 , and 36 left the source surface (based on ballistic back-mapping): (a) 21 Oct. 2007 07:46:17 (CR 2062.53 $\left.=169^{\circ}\right)$, (b) 23 Oct. 2007 03:55:30 $\left(\mathrm{CR} 2062.51=176^{\circ}\right)$, (c) 15 Nov. 2007 21:36:07 (CR $\left.2063.50=180^{\circ}\right)$, (d) 17 Nov. 2007 13:25:30 (CR 2063.45 $\left.=198^{\circ}\right)$, (e) 12 Dec. 2007 20:25:58 (CR 2064.48 = 188 ${ }^{\circ}$, and (f) 14 Dec. 2007 21:35:30 $\left(\mathrm{CR} 2064.45=198^{\circ}\right)$.
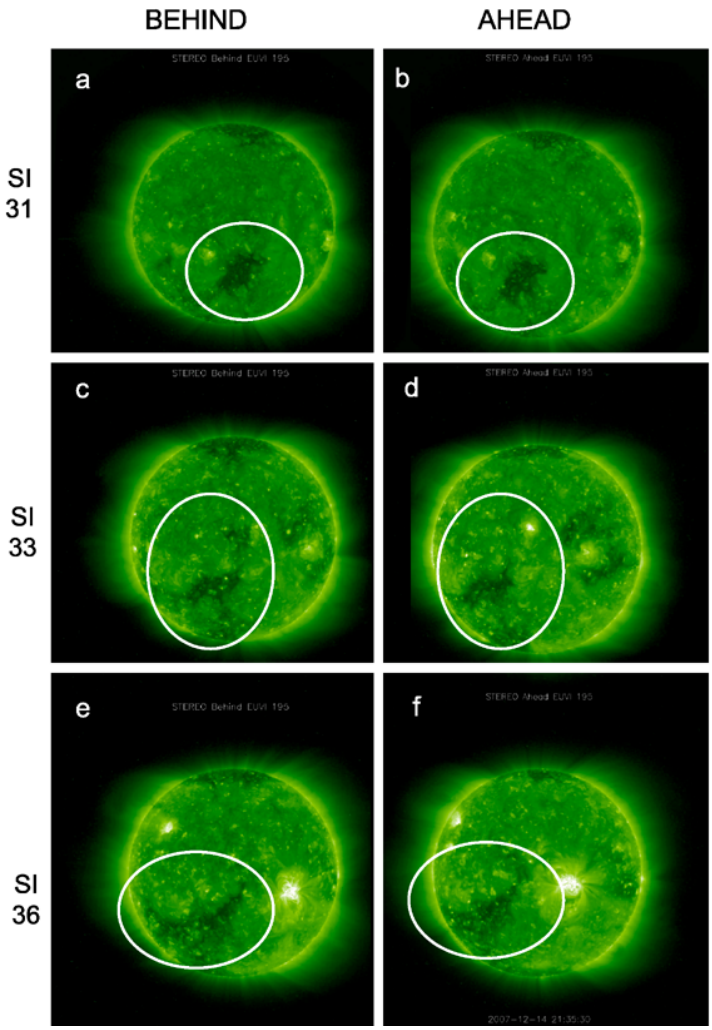

et al. (1976) relationship between maximum solar wind speed and coronal hole area is

$$
V_{\mathrm{SW}}=(80 \pm 2) A+426 \pm 5,
$$

where $V_{\mathrm{SW}}$ is the maximum solar wind speed in $\mathrm{km} \mathrm{s}^{-1}$ and $A$ is the area of the coronal hole within $10^{\circ}$ of the ecliptic plane in units of $10^{10} \mathrm{~km}^{2}$. An increase in maximum solar wind speed of $50 \mathrm{~km} \mathrm{~s}^{-1}$ corresponds to an increase in coronal hole area of $6.25 \times 10^{9} \mathrm{~km}^{2}$, which is about $17 \%$ of the Sun's surface area within $10^{\circ}$ of the ecliptic plane. The SECCHI $195 \AA$ observations in the middle panels of Figure 9 show that the boundaries of the coronal hole have changed between the observations. In addition to the changing boundaries, there is an active region in the image from SECCHI/A that is not seen in the image from SECCHI/B.

In the subsequent Carrington rotation, 2064 (Figure 7), SI no. 36 (the recurrence of no. 33) maps to about $197^{\circ}$ Carrington longitude from both A and B. (This is the same longitude as the mapping from A the previous rotation.) The GONG synoptic map shows that the boundary of the modeled coronal hole has moved to the right (i.e., in the direction of solar rotation) by about $15^{\circ}$. However, the SECCHI synoptic maps in the bottom panel of Figure 7 show that the boundary has not obviously moved from left to right since the previous solar rotation. The SECCHI images in the bottom panels of Figure 9 show qualitative agreement between the two observatories.

Thus we find that our example with the largest time lag discrepancy, SI no. 33, does show a change in the source. However, because it was also a case with a large latitude difference, there is an ambiguity on which is the primary cause. We therefore show one more example to better distinguish between latitudinal dependence and source motion. 
Figure 10 Same as Figure 4, but for Carrington Rotation 2066.

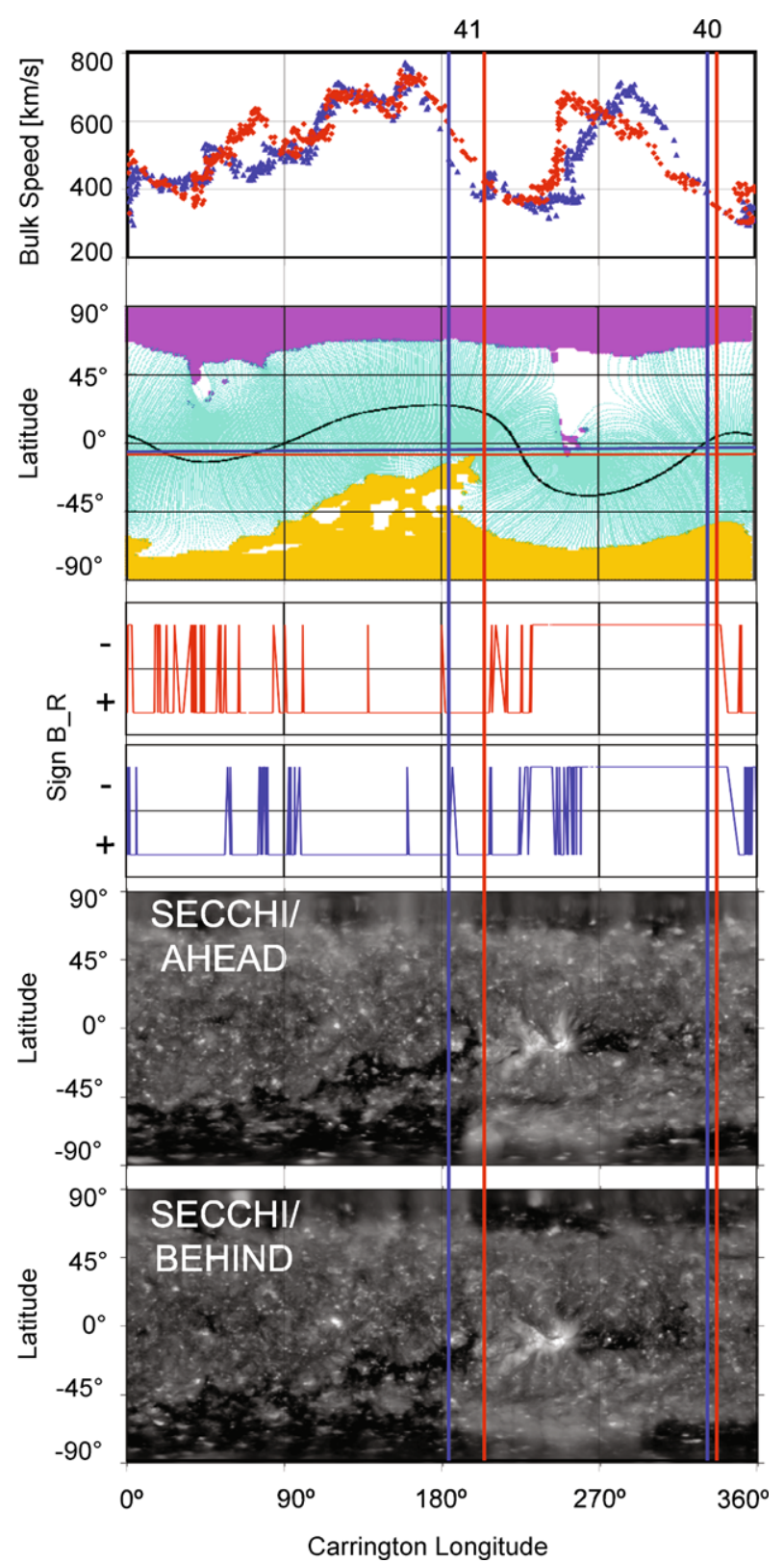

During CR 2066 (February 2008) the radial magnetic polarities line up fairly well between the two spacecraft, as shown in the middle panels of Figure 10. The HCI latitude separation between the spacecraft was less than $3^{\circ}$, and the modeled current sheet was not between the observatories. Even so, the profile of the high-speed stream following SI no. 40 (near $305^{\circ}$ longitude) is obviously different between the two spacecraft. With such a small difference in latitude, evolution of the source between the two observations seems to be the most likely cause for differences in the speed profiles. The SECCHI observations shown in 

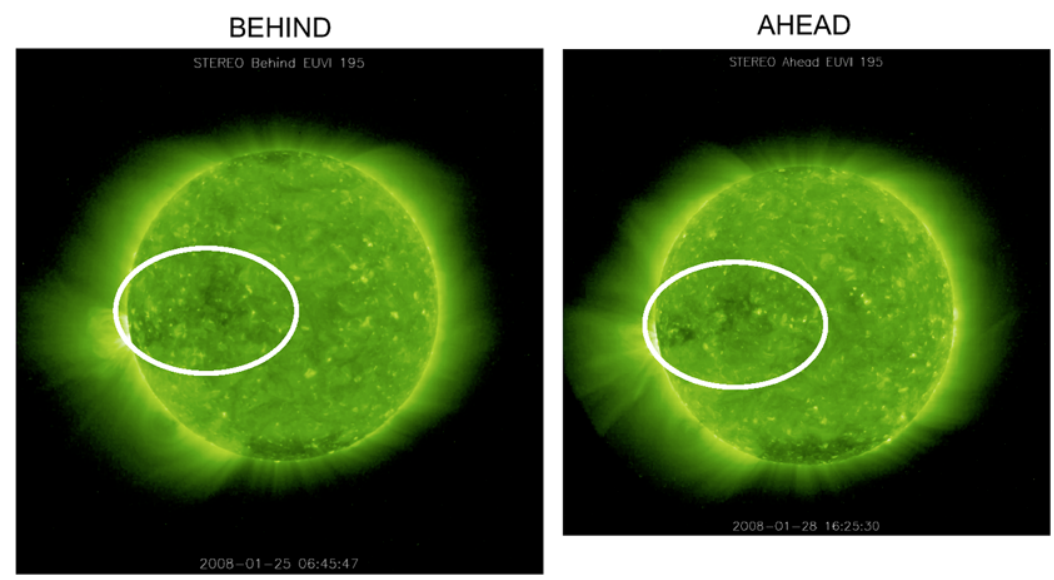

Figure 11 Same as Figure 9, but for SI no. 40.

Figure 11 suggest that the coronal hole is shrinking between the observations by SECCHI/B and SECCHI/A.

\section{Discussion}

Figure 12 shows the difference between expected and observed SI longitude at STEREO/A plotted versus time. The expected longitude was calculated by ignoring latitude separation between the STEREO observatories (as described in Section 3). Through the end of July 2007 , all events except one (no. 14) were measured within $5^{\circ}$ of the expected longitude. In July of 2007, the observed time separation is about 20 hours. From that point on, most SIs are observed within $15^{\circ}$ of the expected longitude. The two events well outside that range (nos. 32 and 33) were both observed during CR 2063, for which qualitative examination of SECCHI images showed coronal hole evolution taking place (Figure 9). Interestingly, the vast majority of cases were observed at earlier longitudes than expected.

One way to obtain a shorter-than-expected time between observations would be if the SIs were propagating slower than expected, leading to an overwound Parker spiral configuration. The phenomenon of SI overwinding was reported by Nolte and Roelof (1973b). Deviations of the interplanetary magnetic field from ideal Archimedean spiral geometry have been reported by Smith and Bieber (1991). Figure 13 shows the effective SI propagation speeds, calculated using Schwenn's technique (Equation (1)), for each of the 41 SIs listed in Table 1 . The effective speeds have been overlaid on a plot of the solar wind speed measured at STEREO/A. We find that through July 2007 there was good agreement between the calculated SI speed and the measured solar wind speed, as expected. However, from August 2007 on, when the longitudinal separation was greater than $22^{\circ}$, and the time separation for SIs was more than a day, we find that the effective propagation speeds of the SIs often fall outside the range of measured solar wind speeds. The effective speeds are in most cases less than the measured solar wind speed, which would be consistent with overwinding and early arrival at STEREO/A. However, if the SI speeds were really significantly less than the solar wind speed, we would observe this independent of the time between the two measurements. In fact, the most accurate measurements of the radial propagation speed are from the time periods when the spacecraft are closest together in longitude. Because we only calculate the 


\section{Difference between Expected and Actual Arrival Longitude at STEREO/A}

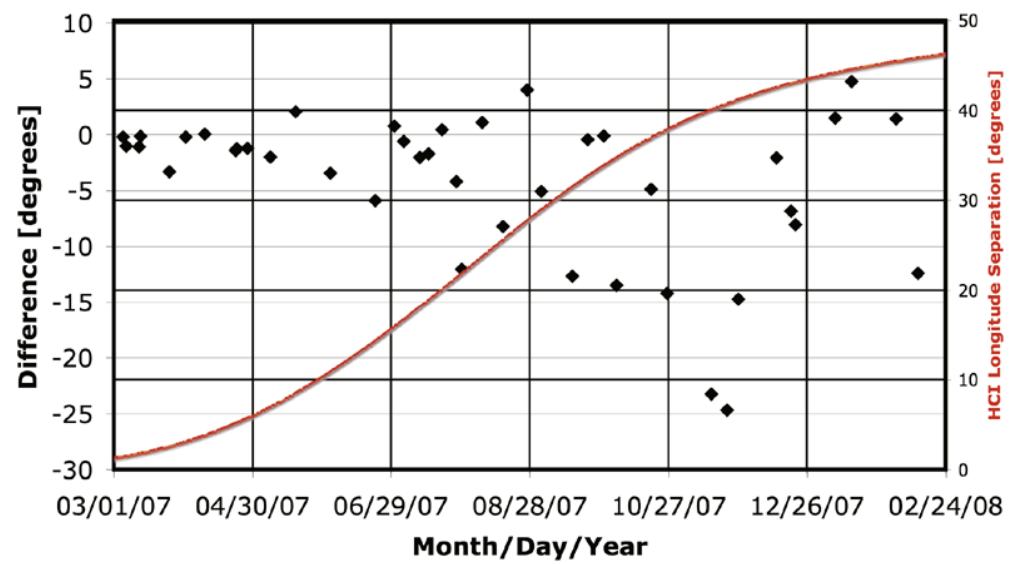

Figure 12 The difference between the calculated and expected observation longitudes versus time, overlaid with a plot of the STEREO HCI longitude separation.

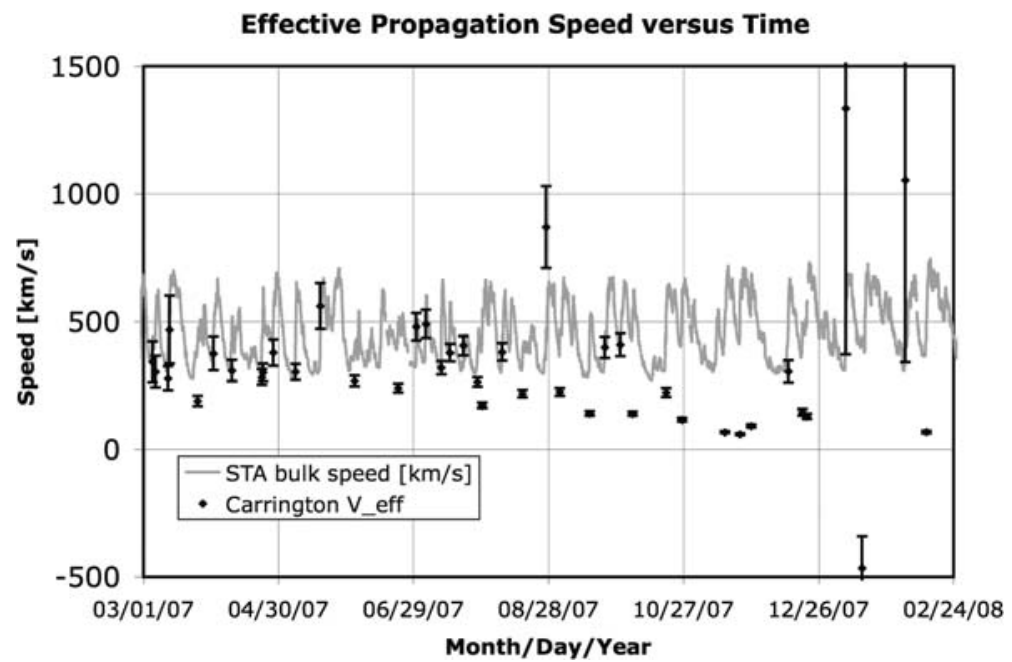

Figure 13 Effective propagation speed versus time. The light gray trace is the solar wind speed measured at STEREO/A. The calculated effective speeds begin to diverge from the measured speeds at the end of July 2007.

low speeds once there is a significant time lag between the two spacecraft measurements; we conclude that this is a result of time or latitude dependence, not a result of a slow SI speed.

\section{Summary and Conclusions}

Forty-one SIs (listed in Table 1) were observed by both PLASTIC/A and PLASTIC/B from March 2007 through February 2008. The effective propagation speeds of each SI were cal- 
culated following the technique of Schwenn (1990). When longitudinal separation between the observatories exceeded about $20^{\circ}$, the calculated effective speeds began to diverge from the measured speeds.

The expected observation longitude at STEREO/A was calculated based on the solar wind speed measured with the SI at STEREO/B. We found that the difference between expected and observed longitudes exceeded $5^{\circ}$ for cases when the effective propagation speed was outside the range of measured speeds. In 32 of 41 cases the SI arrived at STEREO/A earlier than expected. This result is important to forecasting the arrival time of compression regions and high-speed streams at Earth, which are known to cause geomagnetic storms.

In some cases the difference between expected and observed longitudes (and differences in observed speed profiles) can be explained by the difference in spacecraft HCI latitude. However, there are cases where the latitude separation is small and the two observatories see obviously different in situ solar wind speed profiles and different back-mapped Carrington longitudes for the SI. For the cases with the largest differences, evidence for changes in the coronal holes were observed in the EUV images. Thus, both latitude separation and source evolution can be important in determining when a high-speed stream will reach a particular location.

Acknowledgements This work was supported under NASA Contract No. NAS5-00132. This work utilizes data obtained by the Global Oscillation Network Group (GONG) Program, managed by the National Solar Observatory, which is operated by AURA, Inc., under a cooperative agreement with the National Science Foundation. The GONG data were acquired by instruments operated by the Big Bear Solar Observatory, High Altitude Observatory, Learmonth Solar Observatory, Udaipur Solar Observatory, Instituto de Astrofísica de Canarias, and Cerro Tololo Interamerican Observatory. The authors are grateful to the IMPACT team for their data. The SECCHI instrument was constructed by a consortium of international institutions: the Naval Research Laboratory (USA), the Lockheed Martin Solar and Astrophysical Laboratory (USA), the NASA Goddard Space Flight Center (USA), the Max Planck Institut für Sonnensystemforschung (Germany), the Centre Spatial de Liége (Belgium), the University of Birmingham (UK), the Rutherford Appleton Laboratory (UK), the Institut d'Optique (France), and the Institute d'Astrophysique Spatiale (France).

Open Access This article is distributed under the terms of the Creative Commons Attribution Noncommercial License which permits any noncommercial use, distribution, and reproduction in any medium, provided the original author(s) and source are credited.

\section{References}

Burlaga, L.F.: 1974, J. Geophys. Res. 79, 3717.

Galvin, A.B., Kistler, L.M., Popecki, M.A., Farrugia, C.J., Simunac, K.D.C., Ellis, L., Möbius, E., Lee, M.A., Boehm, M., Carroll, J., et al.: 2008, Space Sci. Rev. 136, 437.

Gosling, J.T., Asbridge, J.R., Bame, S.J., Feldman, W.C.: 1978, J. Geophys. Res. 83, 1401.

Gosling, J.T., Bame, S.J., McComas, D.J., Phillips, J.L., Pizzo, V.J., Goldstein, B.E., Neugebauer, M.: 1993, Geophys. Res. Lett. 20, 2789.

Howard, R.A., Moses, J.D., Vourlidas, A., Newmark, J.S., Socker, D.G., Plunkett, S.P., Korendyke, C.M., Cook, J.W., Hurley, A., Davila, J.M., et al.: 2008, Space Sci. Rev. 136, 67.

Kaiser, M.L., Kucera, T.A., Davila, J.M., St. Cyr, O.C., Guhathakurta, M., Christian, E.: 2008, Space Sci. Rev. 136, 5.

Krieger, A.S., Timothy, A.F., Roelof, E.C.: 1973, Solar Phys. 29, 505.

Lee, M.A.: 2000, J. Geophys. Res. 105, 10491.

Leske, R.A., Mewaldt, R.A., Mason, G.M., Cohen, C.M.S., Cummings, A.C., Davis, A.J., Labrador, A.W., Miyasaka, H., Stone, E.C., Wiedenbeck, M.E., von Rosenvinge, T.T.: 2008, In: Li, G., Hu, Q., Verkhoglyadova, O., Zank, G.P., Lin, R.P., Luhmann, J. (eds.) Proceedings of the 7th Annual Astrophysics Conference/Particle Acceleration and Transport in the Heliosphere and Beyond 1039, AIP, New York, 131.

Luhmann, J.G., Curtis, D.W., Schroeder, P., McCauley, J., Lin, R.P., Larson, D.E., Bale, S.D., Sauvaud, J.-A., Aoustin, C., Mewaldt, R.A., et al.: 2008, Space Sci. Rev. 136, 117. 
Mason, G.M., Desai, M.I., Mall, U., Korth, A., Bucik, R., von Rosenvinge, T.T., Simunac, K.D.: 2009, Solar Phys. 256, 393.

Neugebauer, M., Snyder, C.W.: 1966, J. Geophys. Res. 71, 4469.

Neugebauer, M., Forsyth, R.J., Galvin, A.B., Harvey, K.L., Hoeksema, J.T., Lazarus, A.J., Lepping, R.P., Linker, J.A., Mikic, Z., Steinberg, J.T., et al.: 1998, J. Geophys. Res. 103, 14587.

Neugebauer, M., Liewer, P.C., Smith, E.J., Skoug, R.M., Zurbuchen, T.H.: 2002, J. Geophys. Res. 107, 1488.

Newton, H.W., Nunn, M.L.: 1951, Mon. Not. Roy. Astron. Soc. 111, 413.

Nolte, J.T., Roelof, E.C.: 1973a, Solar Phys. 33, 241.

Nolte, J.T., Roelof, E.C.: 1973b, Solar Phys. 33, 483.

Nolte, J.T., Krieger, A.S., Timothy, A.F., Gold, R.E., Roelof, E.C., Vaiana, G., Lazarus, A.J., Sullivan, J.D., McIntosh, P.S.: 1976, Solar Phys. 46, 303.

Schwenn, R.: 1990, In: Schwenn, R., Marsch, E. (eds.) Physics of the Inner Heliosphere I, Springer, Berlin, 99.

Smith, C.W., Bieber, J.W.: 1991, Astrophys. J. 370, 435.

Timothy, A.F., Krieger, A.S., Vaiana, G.S.: 1975, Solar Phys. 42, 135.

Wang, Y.-M., Sheeley, N.R.: 1990, Astrophys. J. 355, 726.

Wang, Y.-M., Sheeley, N.R.: 1993, Astrophys. J. 414, 916.

Wimmer-Schweingruber, R.F., von Steiger, R., Paerli, R.: 1997, J. Geophys. Res. 102, 17407. 